\title{
Reduced fertility and asymptotics of the logistic model
}

\begin{abstract}
This logistic model includes three age groups. Juveniles do not reproduce, and old individuals reproduce at a reduced rate. Pairings between individuals of different fertility rates may lead to multiple equilibria and bistability: the total population converges to different limits depending on its initial size. The behavior is correlated with transition rates from high to low fertility groups and with the frequency of pairing among the various groups of reproduction level. The proportions of adults at equilibrium are roots of a quartic polynomial, alternating sinks and saddles. Necessary and sufficient conditions for the existence of bistability are provided for a simplified model.
\end{abstract}

